\title{
Analyse de la qualité de l'énergie électrique : mesure des signaux déformés
}

\section{Power quality analysis: measurement of distorted signals}

\author{
Patrick ESPEL et André POLETAEFF \\ Laboratoire national de métrologie et d'essais (LNE), 29 Avenue Roger Hennequin, 78197 Trappes Cedex, France, patrick.espel@1ne.fr.
}

\begin{abstract}
Résumé
L'article présente le nouvel étalon primaire du LNE pour les mesures des valeurs efficaces et des taux de distorsion harmonique de signaux périodiques contenant une composante fondamentale à $50 \mathrm{~Hz}$ et des harmoniques quasi-stationnaires jusqu'au rang 50. La méthode de mesure est basée sur l'échantillonnage des signaux par un voltmètre numérique et le traitement des échantillons par transformée de Fourier discrète. La traçabilité aux unités SI est assurée par des comparaisons avec un convertisseur thermique. Les incertitudes types sur la valeur efficace et le taux de distorsion sont respectivement de $22 \mu \mathrm{V} / \mathrm{V}$ et $0,0025 \%$ pour tous les signaux déformés étudiés.
\end{abstract}

MOTS CLÉS : ÉTALON PRIMAIRE, HARMONIQUE QUASISTATIONNAIRE, ÉCHANTILLONNAGE，TEMPS D'INTÉGRATION, CONVERTISSEUR THERMIQUE.

\begin{abstract}
This paper describes a new primary standard at LNE for precision measurement of RMS values and total harmonic distortion of periodic signals containing a fundamental frequency at $50 \mathrm{~Hz}$ and quasistationary harmonics which maximum order is 50. The method is based on the sampling of the signals and the analysis of the samples by discrete Fourier transform. The traceability to the SI units is ensured by comparing the measurements to those obtained using a thermal converter. The standard uncertainty for RMS value and total harmonic distortion are respectively $22 \mu \mathrm{V} / \mathrm{V}$ and $0,0025 \%$ for all the signals studied.
\end{abstract}

KEY WORDS: PRIMARY STANDARD, QUASI-STATIONNARY HARMONIC, SAMPLING TECHNIQUE, APERTURE TIME, THERMAL CONVERTER.

\section{Introduction}

Depuis quelques années, on observe sur les réseaux une augmentation des charges dites non-linéaires liées aux équipements informatiques et à l'électronique de puissance. Ces charges contribuent à la dégradation de la tension d'alimentation et peuvent nuire au bon fonctionnement des équipements et des procédés industriels. Elles génèrent des perturbations dont les principales sont les creux de tension, les harmoniques et inter-harmoniques, les fluctuations de tension (ou Flicker), les déséquilibres de tension, les surtensions (temporaires et transitoires). Afin de caractériser et de quantifier ces perturbations, les industriels ont développés des «mesureurs » de la qualité de l'énergie (qualimètre, flickermètre, perturbographe, analyseur de réseau, analyseur de puissance... ). Les instituts nationaux de métrologie (INM) doivent désormais développer de nouvelles références primaires pour étalonner ces différents appareils. Dans cet article, nous nous intéresserons plus particulièrement aux mesures de la valeur efficace et du taux de distorsion harmonique de signaux périodiques de tension contenant des harmoniques quasi-stationnaires (signaux déformés). Quelques INM disposent déjà d'étalons primaires pour mesurer ces grandeurs $[1,2]$. Ces étalons sont basés sur des techniques numériques qui consistent à échantillonner et quantifier le signal puis à traiter les échantillons ainsi obtenus par transformée de Fourier discrète. Nous avons également développé cette méthode au laboratoire. L'originalité de notre travail porte sur la comparaison des mesures de tension par les techniques d'échantillonnage avec celles réalisées au moyen de convertisseurs thermiques «alternatifcontinu » (AC-DC), afin d'assurer la traçabilité aux unités du SI.

Après avoir présenté succinctement les origines possibles des harmoniques et les principales perturbations 
qu'elles engendrent, nous expliquons les principes de mesure par les techniques numériques et les convertisseurs thermiques. Les principales causes d'erreurs sont énumérées et un bilan d'incertitude est établi. Enfin, les deux méthodes sont comparées pour les mesures des valeurs efficaces de signaux dont le taux de distorsion harmonique varie de $0 \%$ à $84,3 \%$.

\section{Les harmoniques : origines et principales perturbations}

Soit $u(t)$ un signal périodique déformé qui contient des harmoniques quasi-stationnaires jusqu' au rang 50 . Ce signal peut être décomposé en série de Fourier (1) c'est à dire en une somme de composantes sinusoïdales de fréquences $k f_{1}$ :

$$
u(t)=\sum_{k=1}^{50} U_{k} \sin \left(2 \pi k f_{1} t+\varphi_{k}\right),
$$

où $U_{1}$ est la valeur efficace de la composante fondamentale de fréquence $f_{1}$ et $U_{k}$ est la valeur efficace de l'harmonique de fréquence $f_{k}=k f_{1}$.

La valeur efficace $U_{\text {eff }}$ du signal $u(t)$ est alors définie par la relation (2) :

$$
U_{\mathrm{eff}}=\sqrt{U_{1}^{2}+U_{2}^{2}+\ldots+U_{k}^{2}+\ldots} \text {. }
$$

Pour rendre compte de la présence d'harmoniques dans ce signal, on mesure le taux de distorsion harmonique $(T D H)$ qui traduit la déformation du signal par rapport à un signal sinusoïdal de référence. Pour cela, on introduit le «résidu » $r(t)$, signal obtenu lorsque l'on retranche du signal à mesurer sa composante fondamentale, comme exprimé dans la relation (3).

$$
r(t)=\sum_{2}^{\infty} U_{k} \sin \left(2 \pi k f_{k} t+\varphi_{k}\right) .
$$

Le taux de distorsion harmonique $T D H$ est alors défini comme étant le rapport (4) de la valeur efficace du résidu $U_{\text {re }}$ à la valeur efficace de la composante fondamentale $U_{1}$ du signal :

$$
\begin{gathered}
T D H=\frac{U_{\mathrm{re}}}{U_{1}} \\
\text { ou encore, } T D H=\sqrt{\frac{\sum_{k=2} U_{k}^{2}}{U_{1}^{2}}}
\end{gathered}
$$

\subsection{Origines des harmoniques $[3,4]$}

Les harmoniques proviennent principalement de charges non-linéaires dont la caractéristique est d'absorber un courant qui n'a pas la même forme que la tension qui les alimente. Les courants harmoniques circulant à travers les impédances du réseau électrique créent des tensions harmoniques qui peuvent perturber le fonctionnement des autres utilisateurs raccordés à la même source. On distingue deux types de charges :

- les charges industrielles : les équipements d'électronique de puissance (variateurs de vitesse, redresseurs à diodes ou à thyristors, onduleurs... ) et les charges utilisant l'arc électrique (fours à arc, machines à souder, tubes fluorescents... ).

- les charges domestiques munies de convertisseurs ou d'alimentation à découpage : téléviseurs, fours à micro-ondes, plaques à induction, imprimantes, équipements électroménagers...De puissance unitaire bien plus faible que les charges industrielles, leur effet cumulé du fait de leur grand nombre et de leur utilisation simultanée sur de longues périodes, en font des sources de distorsion harmonique importantes.

Quel que soit le type de charges, les niveaux d'harmoniques varient généralement selon le mode de fonctionnement de l'appareil, l'heure de la journée ou encore la saison. Les sources génèrent surtout des harmoniques de rang impair ( $k$ est impair).

\subsection{Principales perturbations provoquées par les courants et tensions harmoniques}

Les tensions et courants harmoniques superposés à l'onde fondamentale conjuguent leurs effets sur les appareils et équipements utilisés. Ces grandeurs harmoniques ont des effets différents selon les récepteurs rencontrés [5] :

- des effets instantanés;

- des effets à terme dus aux échauffements.

Tous ces effets peuvent se traduire par des arrêts des processus industriels, des dysfonctionnements sur les moteurs (vibrations et bruits anormaux), des dysfonctionnements de l'électronique, des perturbations informatiques...

\section{Méthodes et moyens de mesure de la valeur efficace et du taux de distorsion d'un signal déformé}

Les signaux étudiés sont des signaux déformés et périodiques. Ils ont une composante fondamentale «prédominante » à $50 \mathrm{~Hz}$ et un contenu en fréquence limité à l'harmonique de rang 50.

\subsection{Description de l'étalon primaire}

L'étalon primaire est constitué par un voltmètre numérique (DVM) à échantillonnage associé à un système de synchronisation permettant de synchroniser l'échantillonnage avec le signal délivré par la source. L'ensemble est piloté par un ordinateur (fig. 1). Le principe de la mesure consiste à numériser ces signaux puis à traiter les 


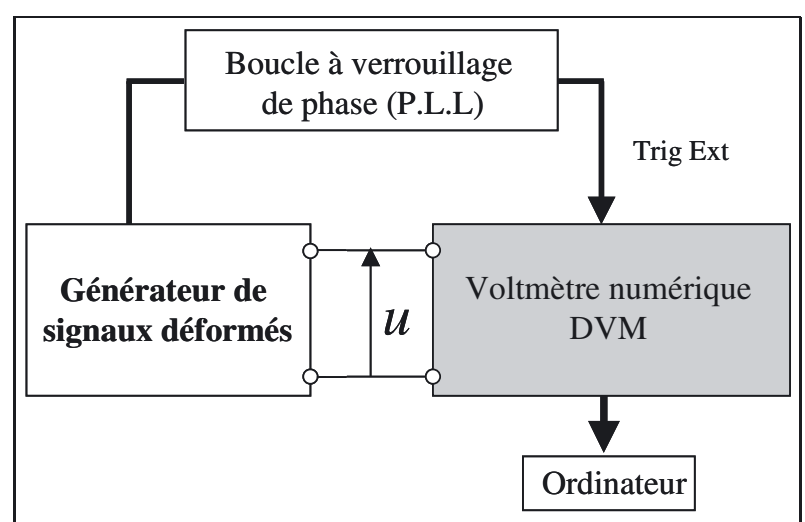

Fig. 1. - Principe de la mesure de signaux déformés avec l'étalon primaire.

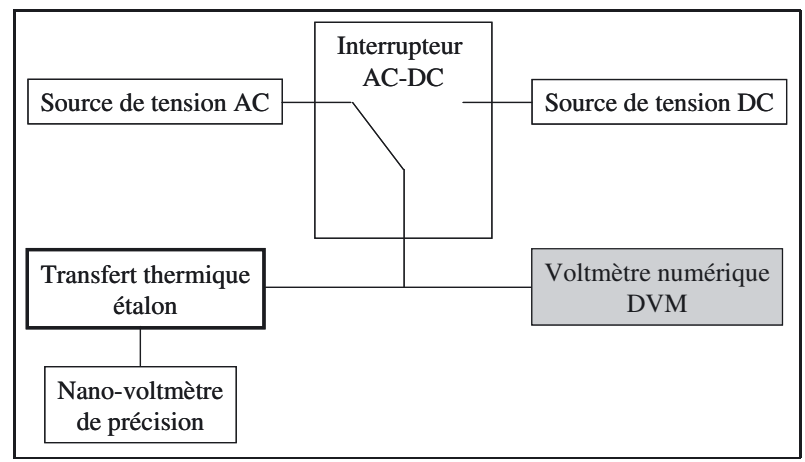

Fig. 2. - Dispositif expérimental pour comparer les mesures RMS par le DVM et le convertisseur thermique.

échantillons obtenus par transformation de Fourier discrète. Les amplitudes $U_{1}$ de la composante fondamentale et $U_{k}$ de chacun des harmoniques sont alors déterminées à partir du spectre d'amplitude. Les valeurs efficaces $U_{\text {eff }}$ des signaux ainsi que leurs taux de distorsion harmonique sont calculés à partir des relations (2) et (4). Pour une plus grande précision des mesures, ce voltmètre est utilisé sur le calibre $1 \mathrm{~V}$ Pour l'analyse de signaux de niveau plus élevé, on insère un diviseur résistif de tension à l'entrée du voltmètre qui engendre une légère dégradation des incertitudes. La numérisation des signaux est effectuée en spécifiant des mesures de tension continue pendant un temps d'intégration $T_{a}$. Les erreurs liées au fait que ce temps d'intégration n'est pas infiniment court ainsi qu'à la bande passante limitée du voltmètre sont calculées et corrigées (voir § 4.1) par le logiciel de pilotage du voltmètre et de traitement des données. Une boucle de réaction à verrouillage de phase (PLL) est utilisée pour générer un signal de cadencement de fréquence $f_{e}$ en phase avec la fréquence fondamentale $f_{1}$ du signal étudié et ainsi éviter les erreurs résultant de l'approximation d'un signal infini par un signal fini.

\subsection{Traçabilité des mesures}

Le dispositif expérimental est représenté sur la figure 2. Le DVM à étalonner et le convertisseur thermique de référence sont connectés en parallèle. Un interrupteur AC-DC permet d'appliquer une tension alternative (AC)

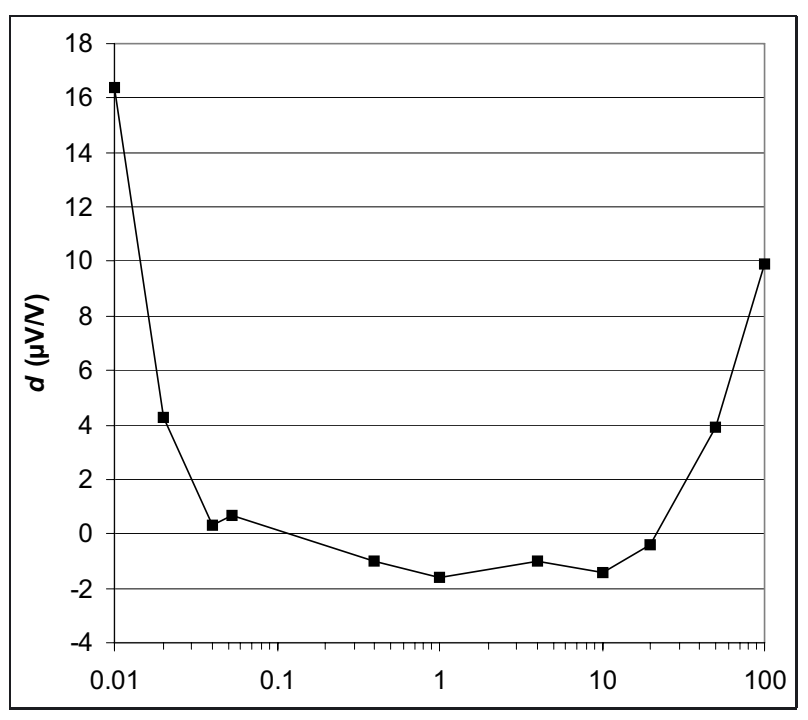

Fig. 3. - Écart de transposition d du convertisseur thermique en fonction de la fréquence.

ou une tension continue (DC). La tension de sortie du convertisseur thermique est mesurée au moyen d'un nanovoltmètre de précision. Le DVM est étalonné périodiquement avec une source de tension continue de référence car il est également utilisé pour mesurer la tension délivrée par la source DC (tension de référence). La séquence $\mathrm{AC}, \mathrm{DC}+, \mathrm{DC}-$ et AC (le signal DC est appliqué dans les deux polarités pour éliminer l'erreur de réversibilité du convertisseur thermique) est alors appliquée et les tensions mesurées par le DVM et le nanovoltmètre de précision sont enregistrées. Le résultat de l'étalonnage $e_{A C}$, grandeur définie comme la différence relative entre les valeurs efficaces du signal mesuré par le DVM en mode échantillonnage et par le convertisseur thermique, est calculé à partir de toutes les données enregistrées :

$$
e_{A C}=\frac{U_{D V M}-U_{C T}}{U_{C T}}
$$

$U_{D V M}$ et $U_{C T}$ sont les valeurs efficaces des signaux de tension $u(t)$ mesurées simultanément par le voltmètre numérique et par le convertisseur thermique.

Le convertisseur thermique est préalablement étalonné sous une tension sinusoïdale de $0,8 \mathrm{~V}$ dans une gamme de fréquences comprises entre $10 \mathrm{~Hz}$ et $100 \mathrm{kHz}$. Son erreur relative, appelée aussi écart de transposition $d$, est représentée sur la figure 3.

Pour connaître l'erreur relative $e_{D V M}$ du DVM sur la mesure de $U_{\text {eff }}$, il suffit donc de retrancher $d$ à $e_{A C}$.

\subsection{Discussion}

Nous avons opté pour une méthode de mesure basée sur les techniques numériques et nous devons comparer cette méthode avec celle utilisant les convertisseurs thermiques afin d'assurer la traçabilité aux unités SI. Si nous n'avons pas fait directement les mesures au moyen des convertisseurs thermiques c'est que, contrairement aux 
convertisseurs thermiques, la méthode numérique permet d'accéder à toutes les grandeurs caractéristiques du signal de tension, c'est-à-dire aux amplitudes et aux phases de la composante fondamentale et de chacun des harmoniques. Elle permet donc de mesurer la valeur efficace totale $U_{\text {eff }}$ du signal mais aussi les valeurs efficaces $U_{k}$ de toutes les composantes de ce signal et son taux de distorsion harmonique $T D H$ alors que les convertisseurs thermiques sont limités à la mesure de $U_{\text {eff }}$.

\section{Analyse des différentes sources d'erreurs et bilan d'incertitude}

\subsection{Principales sources d'erreurs}

Les principales sources d'erreurs (celles qui ont une influence significative sur l'exactitude de nos mesures) sont liées à l'utilisation du DVM en mode échantillonnage par lecture de tension continue et à sa bande passante limitée. Elles ont été évaluées par le calcul et sont présentées dans les paragraphes suivants.

\subsubsection{Erreur sur la détermination de $U_{k}$ due à la limitation de bande passante du DVM}

Le DVM est utilisé sur le calibre $1 \mathrm{~V}$ et sa bande passante est limitée à $150 \mathrm{kHz}$. Cette limitation introduit une erreur $e_{B P}$ sur la mesure de la valeur efficace des différentes composantes des signaux de tension $u(t)$.

En modélisant la bande passante par un filtre passebas du deuxième ordre [6], on montre que cette erreur s'écrit sous la forme (6) :

$$
\begin{aligned}
e_{B P, k} & =\left(\frac{\Delta U}{U}\right)_{B P, k} \\
& =\frac{1}{\sqrt{1+7\left(2 \pi f_{k} R C\right)^{2}+\left(2 \pi f_{k} R C\right)^{4}}}-1
\end{aligned}
$$

$f_{k}$ est la fréquence du signal, $R$ et $C$ sont la résistance et la capacité du filtre de valeurs respectives $5 \mathrm{k} \Omega$ et $82 \mathrm{pF}$.

L'erreur $e_{B P}$ est représentée sur la figure 4 en fonction de la fréquence $f_{k}$ de toutes les composantes du signal.

\subsection{2. $\quad$ Erreur sur la détermination de $U_{k}$ due au temps d'intégration du DVM}

Lorsque l'opération d'échantillonnage est réalisée avec une impulsion infiniment brève (cas théorique), le signal échantillonné s'écrit sous la forme :

$$
\hat{u}(t, N)=\sum_{k=0}^{N-1} u\left(k T_{e}\right) \cdot \delta\left(t-k T_{e}\right)
$$

où $\sum_{k=0}^{N-1} \delta\left(t-k T_{e}\right)$ est une suite périodique de distribution de Dirac (peigne de Dirac), de période $T_{e}$.

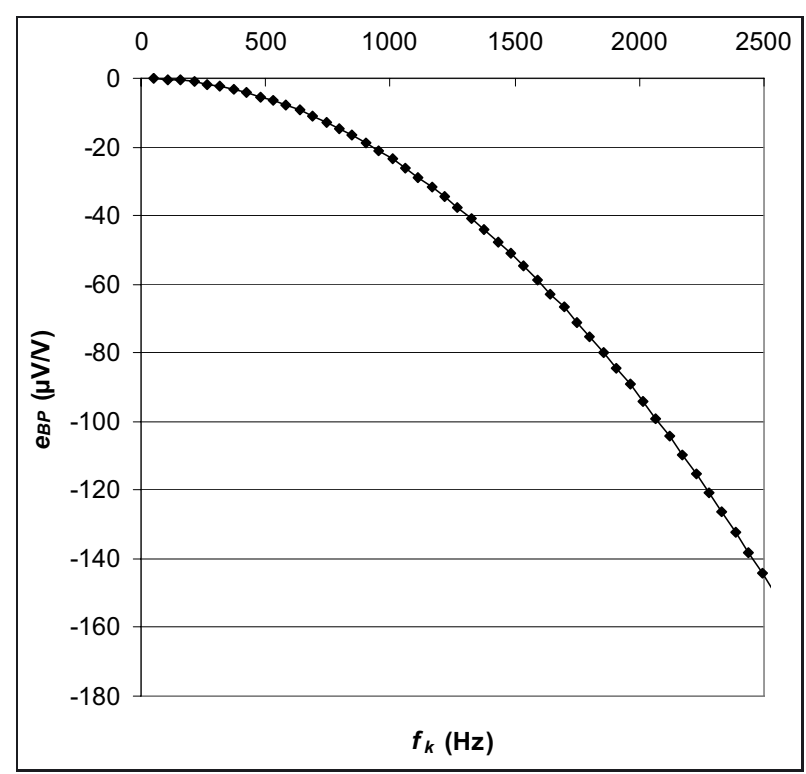

Fig. 4. - Erreur $e_{B P}$ en fonction de la fréquence $f_{k}$ des composantes du signal.

Sa transformée de Fourier est alors égale à :

$$
\begin{aligned}
& \sum_{k=0}^{N-1} u\left(k T_{e}\right) \cdot \exp \left(-2 \pi j f k T_{e}\right), \text { ou encore } \\
& U(f) * F_{e} \cdot \sum_{n=-\infty}^{+\infty} \delta\left(f-n F_{e}\right) .
\end{aligned}
$$

En pratique, l'opération d'échantillonnage n'est pas réalisée avec une impulsion infiniment brève. Le voltmètre établit une moyenne sur le signal d'entrée pendant une durée $T_{a}$ et chaque échantillon $u\left(k T_{e}\right)$ s'exprime sous la forme (9) :

$$
u\left(k T_{e}\right)=\frac{1}{T_{a}} \int_{k T_{e}-\frac{T_{a}}{2}}^{k T_{e}+\frac{T_{a}}{2}} u(t) d t=\left.\frac{1}{T_{a}} u(t) * \pi_{\frac{T_{a}}{2}}(t)\right|_{t=k T_{e}} .
$$

Le signal échantillonné s'écrit alors (10) :

$$
\hat{u}(t)=\frac{1}{T_{a}}\left[u(t) * \pi_{\frac{T_{a}}{2}}(t)\right] \sum_{k=-\infty}^{+\infty} \delta\left(t-k T_{e}\right)
$$

et sa transformée de Fourier est égale à (11) :

$$
\left[U(f) \frac{\sin \left(\pi f T_{a}\right)}{\pi f T_{a}} \exp \left(-2 \pi j f \frac{T_{a}}{2}\right)\right] * F_{e} \cdot \sum_{n=-\infty}^{+\infty} \delta\left(f-n F_{e}\right) .
$$

Lorsque $T_{a}$ est non nulle (cas réel), tout se passe donc comme si $U(f)$ de l'équation (8) était remplacé par $U^{\prime}(f)$ telle que :

$$
U^{\prime}(f)=U(f) \frac{\sin \left(\pi f T_{a}\right)}{\pi f T_{a}} \exp \left(-2 \pi j f \frac{T_{a}}{2}\right) .
$$




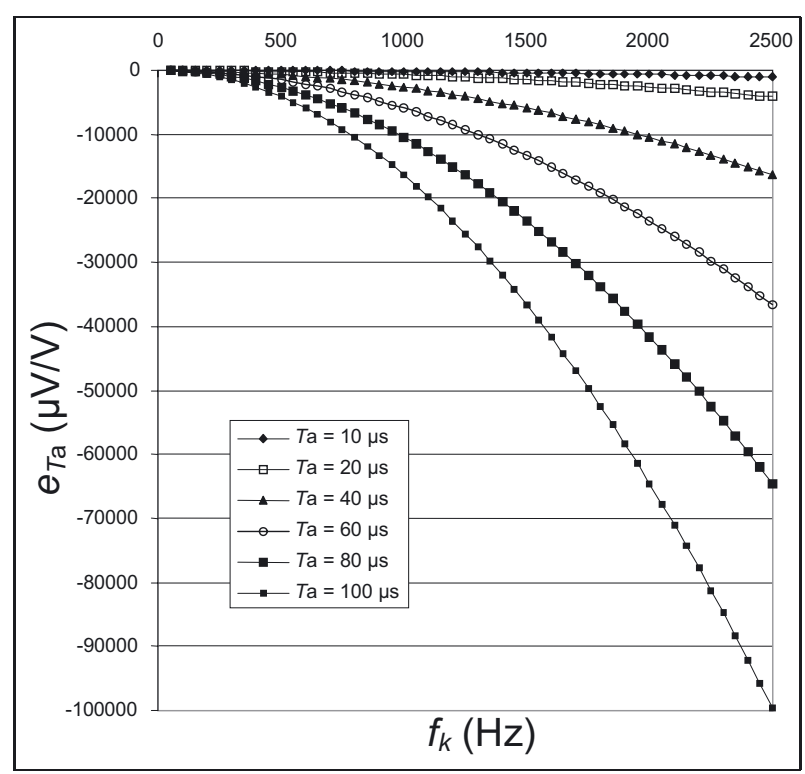

Fig. 5. - Erreur $e_{T_{a}}$ en fonction de la fréquence $f_{k}$ des composantes du signal, pour différentes valeurs de $T_{a}$ comprises entre $10 \mu$ s et $100 \mu \mathrm{s}$.

Autrement dit, le module du spectre est filtré par le sinus cardinal de $\left(\pi f T_{a}\right)$ et l'erreur relative résultante $e_{T_{a}}$ sur la mesure de $U_{\text {eff }}$ est alors définie par :

$$
e_{T_{a}, k}=\left(\frac{\Delta U}{U}\right)_{T_{a}, k}=\operatorname{sinc}\left(\pi f_{k} T_{a}\right)-1
$$

Cette erreur est nulle lorsque l'opération d'échantillonnage est réalisée avec une impulsion infiniment brève $\left(T_{a}=0\right)$ quelles que soient les valeurs de la fréquence $f_{k}$. Pour un $T_{a}$ donné, elles augmentent considérablement avec le rang de l'harmonique $f_{k}$ (fig. 5). Par exemple, pour $T_{a}=60 \mu \mathrm{s}$ et $f_{50}=2500 \mathrm{~Hz}$, l'erreur est de $-36602 \mu \mathrm{V} / \mathrm{V}$.

Toutes les erreurs $e_{B P}$ et $e_{T_{a}}$ sont systématiques et sont corrigées pour chacun des harmoniques de fréquence $f_{k}$ présents dans le signal.

\subsection{Mesures préliminaires en régime sinusoïdal}

Une étude complète [7] a été réalisée sur la plage de fréquences comprises entre $20 \mathrm{~Hz}$ et $400 \mathrm{~Hz}$ pour des signaux sinusoïdaux ou présentant un $T D H$ inférieur à $0,1 \%$. L'influence des différents paramètres de numérisation a été étudié. Ainsi, pour une fréquence d'échantillonnage $f_{e}$ voisine de $10 \mathrm{kHz}$ et des temps d'intégration $T_{a}$ compris entre $50 \mu \mathrm{s}$ et $90 \mu \mathrm{s}$, l'erreur relative $e_{D V M} \mathrm{n}$ 'excède pas $5 \times 10^{-6}$.

Pour étudier des signaux déformés dont la composante fondamentale "prédominante » est à $50 \mathrm{~Hz}$ et le contenu harmonique est limité au rang 50, il est au préalable nécessaire de compléter l'étude précédente et d'étudier le comportement du DVM lorsqu'on lui applique un signal sinusoïdal dont la fréquence varie de $50 \mathrm{~Hz}$ à

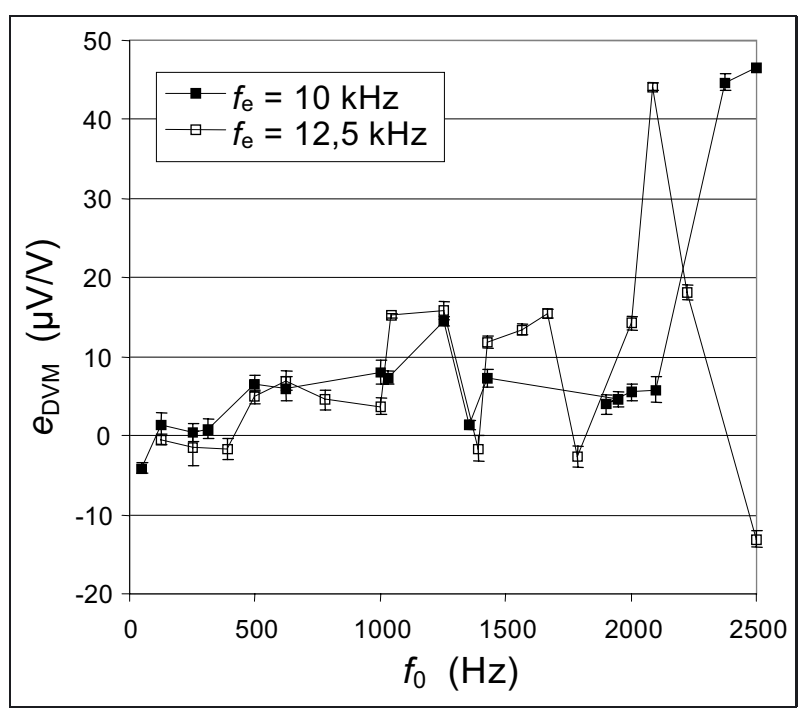

Fig. 6. - Erreur relative $e_{D V M}$ pour des signaux sinusoïdaux dont la fréquence varie de $50 \mathrm{~Hz}$ à $2500 \mathrm{~Hz}$.

$50 \times 50 \mathrm{~Hz}$, soit $2500 \mathrm{~Hz}$. L'erreur relative $e_{D V M}$ du voltmètre sur la mesure de $U_{\text {eff }}$ est représentée sur la figure 6 pour des valeurs de $f_{e}$ égales à $10 \mathrm{kHz}$ et $12,5 \mathrm{kHz}$ et un temps d'intégration fixe de $60 \mu$ s. Elle tient compte des corrections apportées au voltmètre en raison de sa limitation de bande passante et de son temps d'intégration nonnul. Sur toute la plage de fréquence, cette erreur n'excède pas $50 \times 10^{-6}$.

\subsection{Bilan d'incertitude}

\subsubsection{Incertitude sur la mesure de $U_{\mathrm{eff}}$}

En appliquant la loi de propagation des incertitudes à la relation (2), on montre que l'incertitude type relative sur la mesure de $U_{\text {eff }}$ s'écrit sous la forme :

$$
\left(\frac{\sigma_{U_{\text {eff }}}}{U_{\text {eff }}}\right)^{2}=\sum_{k=1}^{50}\left(\frac{U_{k}}{U_{\text {eff }}}\right)^{4} \times\left(\frac{\sigma_{U_{k}}}{U_{k}}\right)^{2}
$$

ou encore,

$$
\left(\frac{\sigma_{U_{\mathrm{eff}}}}{U_{\mathrm{eff}}}\right)^{2}=\sum_{k=1}^{50}\left(\frac{U_{k}}{U_{\mathrm{eff}}}\right)^{2} \times\left(\frac{\sigma_{U_{k}}}{U_{\mathrm{eff}}}\right)^{2} .
$$

Posons

$$
A_{k}=\left(\frac{\sigma_{U_{k}}}{U_{\mathrm{eff}}}\right)=\left(\frac{\sigma_{U_{k}}}{U_{k}}\right) \times\left(\frac{U_{k}}{U_{\mathrm{eff}}}\right) .
$$

Une valeur majorée de $\left(\frac{\sigma_{U_{k}}}{U_{k}}\right)$ est $50 \times 10^{-6}$. D'autre part, quel que soit le rang de l'harmonique, le rapport $\left(\frac{U_{k}}{U_{\text {eff }}}\right)$ n'excède pas $50 \%$ (tab. 1). La valeur maximale de $A_{k}$ est donc de $25 \times 10^{-6}$.

La relation (14) peut alors s'écrire :

$$
\left(\frac{\sigma_{U_{\mathrm{eff}}}}{U_{\mathrm{eff}}}\right)^{2} \leq\left(25 \times 10^{-6}\right)^{2} \times \sum_{k=1}^{50}\left(\frac{U_{k}}{U_{\mathrm{eff}}}\right)^{2} .
$$


Tableau 1

Composition spectrale des différents signaux étudiés.

\begin{tabular}{|c|l|c|}
\hline Signal & Composition harmonique & TDH $(\%)$ \\
\hline 1 & $U_{1}=1 \mathrm{~V}$ & 0 \\
\hline 2 & $U_{1}=1 \mathrm{~V}, U_{6}=0,01 \mathrm{~V}$ & 1 \\
\hline 3 & $U_{1}=1 \mathrm{~V}, U_{3}=0,01 \mathrm{~V}, U_{10}=0,05 \mathrm{~V}$ & 5,1 \\
\hline 4 & $U_{1}=1 \mathrm{~V}, U_{5}=U_{15}=0,05 \mathrm{~V}$ & 7,9 \\
& $U_{30}=U_{40}=U_{50}=0,02 \mathrm{~V}$ & \\
\hline 5 & $U_{1}=1 \mathrm{~V}, U_{20}=0,1 \mathrm{~V}$ & 10 \\
\hline 6 & $U_{1}=1 \mathrm{~V}, U_{3}=U_{25}=U_{45}=0,1 \mathrm{~V}$ & 17,3 \\
\hline 7 & $U_{1}=1 \mathrm{~V}, U_{2}=U_{3}=0,1 \mathrm{~V}$ & 25,1 \\
& $U_{4}=0,05 \mathrm{~V}, U_{5}=0,02 \mathrm{~V}, U_{50}=0,2 \mathrm{~V}$ & \\
\hline 8 & $U_{1}=1 \mathrm{~V}, U_{50}=0,3 \mathrm{~V}$ & 30 \\
\hline 9 & $U_{1}=0,9 \mathrm{~V}, U_{2}=U_{36}=0,2 \mathrm{~V}$, & 35,1 \\
& $U_{34}=U_{35}=0,1 \mathrm{~V}$ & 42,4 \\
\hline 10 & $U_{1}=1 \mathrm{~V}, U_{3}=U_{4}=0,3 \mathrm{~V}$ & 49,2 \\
\hline 11 & $U_{1}=0,8 \mathrm{~V}, U_{2}=0,25 \mathrm{~V}, U_{9}=0,25 \mathrm{~V}$ & 51,5 \\
& $U_{22}=U_{41}=U_{47}=0,1 \mathrm{~V}$ & \\
\hline 12 & $U_{1}=0,8 \mathrm{~V}, U_{20}=0,3 \mathrm{~V}$, & 58,6 \\
& $U_{36}=U_{50}=0,2 \mathrm{~V}$ & \\
\hline 13 & $U_{1}=0,8 \mathrm{~V}, U_{48}=0,2 \mathrm{~V}$, & \\
& $U_{49}=U_{50}=0,3 \mathrm{~V}$ & 72,3 \\
\hline 14 & $U_{1}=0,8 \mathrm{~V}, U_{2}=U_{4}=U_{6}=U_{18}=$ & 63,7 \\
& $U_{25}=U_{26}=0,2 \mathrm{~V}, U_{27}=U_{28}=0,1 \mathrm{~V}$ & \\
\hline 15 & $U_{1}=0,7 \mathrm{~V}, U_{2}=0,3 \mathrm{~V}, U_{13}=0,28 \mathrm{~V}$ & 64 \\
& $U_{34}=0,18 \mathrm{~V}$ & 79,9 \\
\hline 16 & $U_{1}=0,65 \mathrm{~V}, U_{22}=U_{34}=0,3 \mathrm{~V}$ & \\
\hline 17 & $U_{40}=0,2 \mathrm{~V}$ & \\
& $U_{1}=0,65 \mathrm{~V}, U_{4}=U_{5}=U_{6}=U_{7}=$ & 72,2 \\
& $0,2 \mathrm{~V}$, & \\
\hline 18 & $U_{14}=U_{15}=U_{16}=U_{17}=U_{29}=0,1 \mathrm{~V}$ & \\
\hline 19 & $U_{1}=0,65 \mathrm{~V}, U_{22}=0,4 \mathrm{~V}, U_{34}=0,3 \mathrm{~V}$ & \\
& $U_{40}=U_{50}=0,1 \mathrm{~V}$ & \\
\hline 20 & $U_{4}=0,65 \mathrm{~V}, U_{2}=0,3 \mathrm{~V}, U_{3}=0,25 \mathrm{~V}$ & $U_{1}=0,65 \mathrm{~V}, U_{2}=U_{3}=0,3 \mathrm{~V}$ \\
& $U_{4}=U_{5}=U_{6}=0,2 \mathrm{~V}$ & \\
\hline
\end{tabular}

Or, par définition de la valeur efficace d'un signal déformé de la forme $u(t)$, défini par (1), on a :

$$
\sum_{k=1}^{50}\left(\frac{U_{k}}{U_{\mathrm{eff}}}\right)^{2}=1
$$

La valeur maximale de l'incertitude type relative sur la valeur efficace du signal est finalement égale à $25 \times 10^{-6}$.

\subsubsection{Incertitude sur la mesure de TDH}

En appliquant la loi de propagation des incertitudes à la relation (4) et en tenant compte des corrélations entre les grandeurs $U_{1}$ et $U_{\text {eff }}$, l'incertitude type absolue sur la mesure du taux de distorsion $T D H$ s'écrit sous la forme :

$$
\begin{aligned}
\sigma_{T D H}= & \frac{\left(1+T D H^{2}\right)}{T D H} \\
& \times \sqrt{\left(\frac{\sigma_{U_{1}}}{U_{1}}\right)^{2}\left(1-\frac{2}{1+T D H^{2}}\right)+\left(\frac{\sigma_{U_{\text {eff }}}}{U_{\text {eff }}}\right)^{2}} .
\end{aligned}
$$

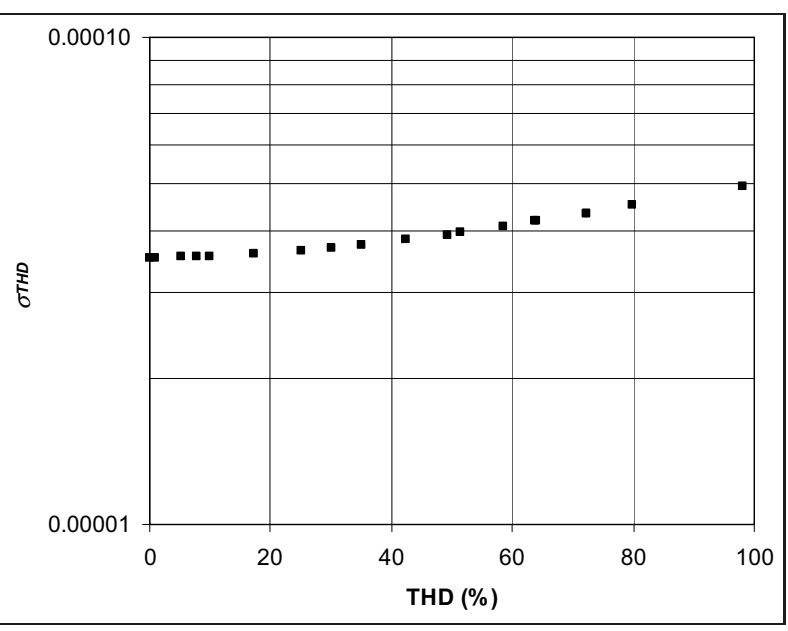

Fig. 7. - Incertitude type associée à la mesure du TDH en fonction des TDH des signaux étudiés.

Cette incertitude est calculée en fonction des taux de distorsion des différents signaux que nous étudions par la suite (tab. 1). Les résultats sont présentés sur la figure 7. Pour tous les signaux dont les $T D H$ varient de $0,1 \%$ à $100 \%$, l'incertitude type reste inférieure à $0,005 \%$. Ce résultat a été obtenu en majorant l'incertitude calculée au paragraphe 4.3.1 par :

$$
\operatorname{Maj}\left(\frac{\sigma_{U_{\mathrm{eff}}}}{U_{\mathrm{eff}}}\right)=\operatorname{Maj}\left(\frac{\sigma_{U_{1}}}{U_{1}}\right)=25 \times 10^{-6} .
$$

\section{Résultats expérimentaux}

\subsection{Influence du temps d'intégration $T_{a}$ du DVM}

Les signaux de tension $u(t)$ étudiés sont les suivants :

- un signal sinusoïdal (ou présentant un $T D H$ inférieur à $0,1 \%$ ) de fréquence $50 \mathrm{~Hz}$,

- trois signaux déformés avec une composante fondamentale à $50 \mathrm{~Hz}$ et un harmonique de rang 10 et d'amplitude variable (signal $1: U_{1}=0,05 \mathrm{~V}$, signal 2 : $U_{2}=0,075 \mathrm{~V}$, signal $\left.3: U_{3}=0,1 \mathrm{~V}\right)$.

Lors de l'opération de numérisation, le DVM prélève 1024 échantillons du signal $u(t)$ sur 5 périodes. La fréquence d'échantillonnage $f_{e}$ qui vérifie la relation (imposée par la PLL)

$$
f_{e}=\frac{N}{M} f_{0}
$$

est alors égale à 10,24 kHz.

Cette fréquence d'échantillonnage est maintenue constante lors des mesures alors que le temps d'intégration $T_{a}$ prend des valeurs différentes, comprises entre $10 \mu$ s et $80 \mu \mathrm{s}$. Les variations de l'erreur relative $e_{D V M}$ sont présentées sur la figure 8 en fonction du temps d'intégration $T_{a}$. L'allure des courbes est la même pour tous les signaux étudiés. Les valeurs absolues de $e_{\mathrm{DVM}}$ fluctuent entre $1 \mu \mathrm{V} / \mathrm{V}$ et $12 \mu \mathrm{V} / \mathrm{V}$ en fonction du temps 


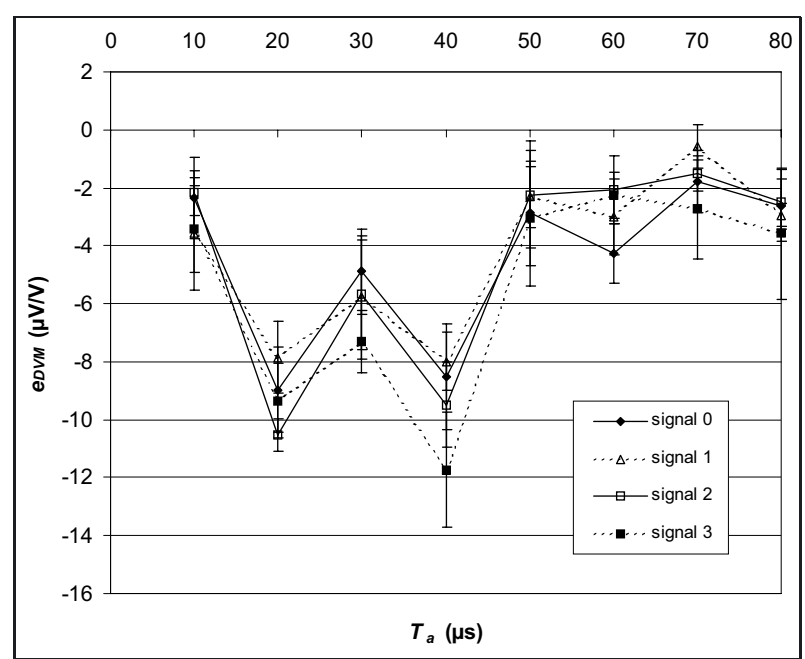

Fig. 8. - Influence du temps d'intégration $T_{a}$ du DVM sur la mesure de $U_{\text {eff }}$.

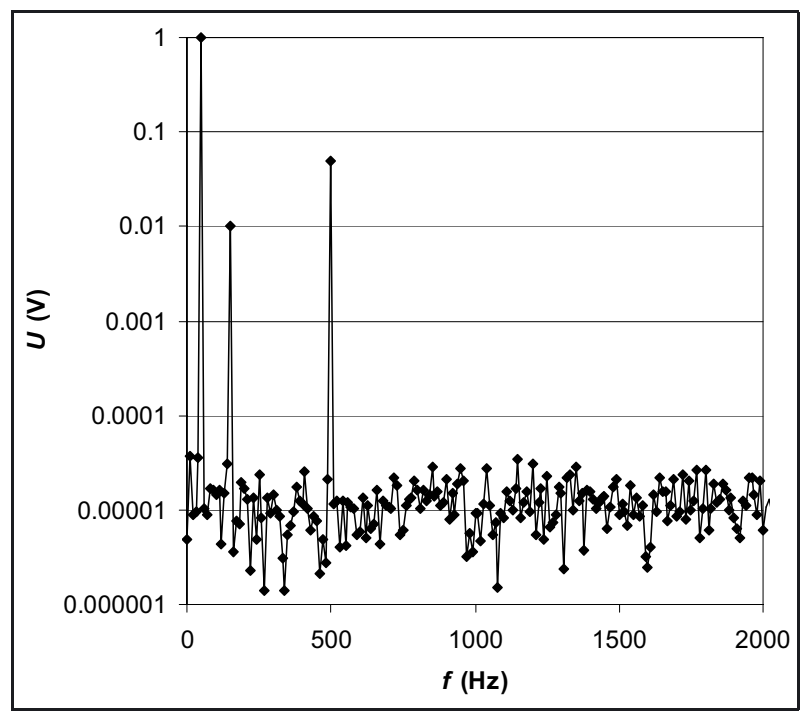

Fig. 9. - Exemple de spectre d'amplitude (le signal étudié est le signal 3).

d'intégration $T_{a}$ choisi et du signal considéré. Les erreurs les plus faibles (inférieures à $4 \mu \mathrm{V} / \mathrm{V}$ ) sont obtenues pour des valeurs de $T_{a}$ comprises entre $50 \mu$ s et $80 \mu \mathrm{s}$. Il conviend donc de choisir préférentiellement une de ces valeurs pour les mesures.

\subsection{Influence du $T D H$ sur la mesure de $U_{\text {eff }}$}

Lors de l'opération de numérisation, la fréquence d'échantillonnage $f_{e}$ est égale à $10,24 \mathrm{kHz}$ et le temps d'intégration choisi est de $60 \mu \mathrm{s}$. On mesure les valeurs efficaces d'un signal de tension sinusoïdal et de 19 signaux déformés dont les $T D H$ s'échelonnent entre $1 \%$ et $84,3 \%$. Les amplitudes de la composante fondamentale et des harmoniques de chacun de ces signaux sont détaillées dans le tableau 1.

Un exemple de spectre d'amplitude est donné sur la figure 9. Il présente trois pics qui correspondent à la composante fondamentale à $50 \mathrm{~Hz}$ et deux harmoniques

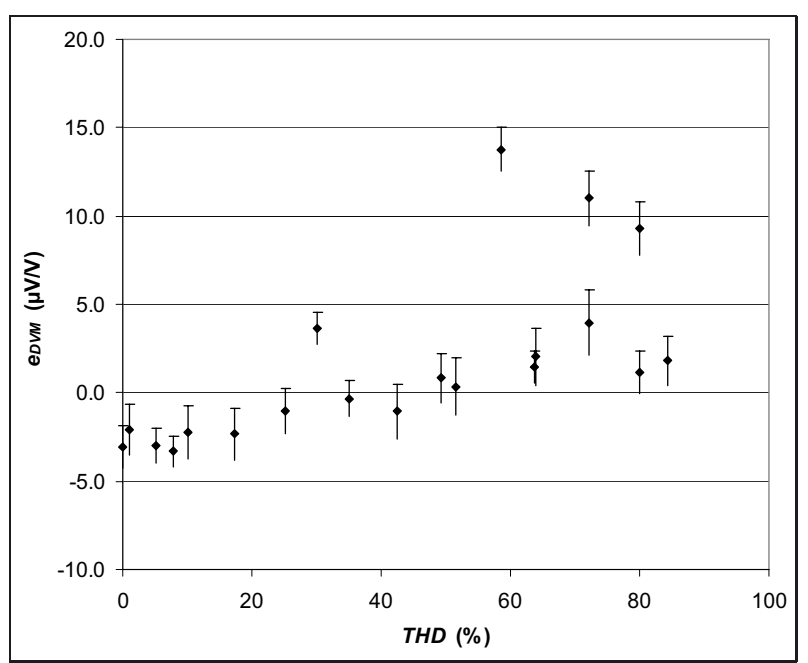

Fig. 10. - Comparaison entre la technique d'échantillonnage et le convertisseur thermique pour la mesure de la valeur efficace $U_{\text {eff }}$ de signaux déformés.

de rangs 3 et 10 (signal 3 ). Le niveau de «zéro » (en moyenne de l'ordre de $1 \times 10^{-5}$ ) est essentiellement dû au bruit de quantification. En effet, la quantification impose théoriquement un rapport signal sur bruit (en décibel, dB) sensiblement égal à six fois le nombre de bits de quantification [6]. Dans notre configuration expérimentale, le codage se fait sur 18 bits. Le rapport signal sur bruit est donc de $108 \mathrm{~dB}$, soit un niveau de bruit de l'ordre de $10^{-5} \mathrm{~V}$ à $10^{-6} \mathrm{~V}$ pour un signal d'amplitude $1 \mathrm{~V}$. Ce calcul est en bon accord avec les résultats expérimentaux présentés en figure 9 .

Les résultats de la comparaison entre la technique d'échantillonnage et le convertisseur thermique sont présentés sur la figure 10, pour la mesure de la valeur efficace $U_{\text {eff }}$. Pour tous les signaux étudiés, l'écart relatif n'excéde pas $15 \times 10^{-6}$ et est le plus souvent de 2 à $3 \mu \mathrm{V} / \mathrm{V}$. L'écart de transposition du convertisseur thermique sur la plage de fréquence correspondant à l'étendue spectrale du signal étant toujours inférieur à $2 \mu \mathrm{V} / \mathrm{V}$, l'erreur du voltmètre est de l'ordre d'une quinzaine de $\mu \mathrm{V} / \mathrm{V}$ dans les cas les plus défavorables et le plus souvent inférieure à $5 \mu \mathrm{V} / \mathrm{V}$, ce qui est en parfait accord avec le calcul d'incertitude du paragraphe 4.3.1.

\section{Conclusion}

Un nouvel étalon primaire a été développé au LNE pour la mesure de la valeur efficace $U_{\text {eff }}$ et du taux de distorsion harmonique $T D H$ de signaux déformés dont la composante fondamentale est à $50 \mathrm{~Hz}$ et le contenu harmonique est limité au rang 50. La méthode basée sur la numérisation du signal et le traitement de ses échantillons par transformation de Fourier discrète permet de mesurer ces grandeurs respectivement avec des incertitudes types de $22 \mu \mathrm{V} / \mathrm{V}$ et $0,0025 \%$ pour tous les signaux étudiés. La traçabilité des mesures aux unités SI est assurée par une comparaison de la méthode numérique à une méthode thermique basée sur l'utilisation de convertisseurs 
thermiques. L'écart relatif entre les deux méthodes n'excède pas $15 \times 10^{-6}$.

\section{Références}

[1] ARseneau R., Sutherland M., Zelle J. et SVEnsson S., "Comparison of non-sinusoidal calibration systems at NRC Canada and SP Sweden", IEEE Trans. Instr. And Meas., 50, 2001, 275-277.

[2] SVENSSON S., "A precision wattmeter for non-sinusoidal conditions", Technical Report No. 223L, Chalmers university of Technology, Göteborg, Suède.

Article reçu le 19 septembre 2010, version révisée reçue le 23 mai 2011.
[3] R.CALVAS, «Les perturbations électriques en BT», Cahier technique $n^{\circ}$ 141, Schneider Electric.

[4] Ferracci Ph., «La qualité de l'énergie électrique », Cahier technique $n^{\circ}$ 199, Schneider Electric.

[5] Peyrucat J-F., «Les analyseurs de la qualité du réseau électrique », Mesures, 770, décembre 2004, www. mesures.com.

[6] KURTEN IHLENFELD W.G., "Maintenance and traceability of AC voltages by synchronous digital synthesis and sampling”, PTB-E-75, Braunschweig, août 2001.

[7] Espel P., PoletaefF A. et Bounouh A., "Characterization of analogue-to-digital converters of a commercial digital voltmeter in the $20 \mathrm{~Hz}$ to $400 \mathrm{~Hz}$ frequency range.”, Metrologia, 46, 2009, 578-584. 